\title{
A NEW PISTON VENTILATOR FOR USE IN RESPIRATORY STUDIES
}

\author{
G.A. VOLGYESI AND \\ H.I.A. NISBET, M.B., CH.B., F.E.A.R.C.S.(ENG.), C.R.C.P.(C)
}

SPECral EQUIPMENT is needed to study the effect of long-term positive pressure ventilation on functional residual capacity (FRC), oxygen consumption $\left(\dot{\mathrm{vO}}_{2}\right.$ ) and other respiratory parameters. The apparatus must be small, portable and able to ventilate the patient in closed circuit during FRC measurement, and it must provide various tidal volumes and minute ventilation for patients of different sizes.

The operator of the equipment must be able to stop ventilation at any chosen point on the respiratory cycle and restart it from the same point to allow the switching of the patient in and out of the closed circuit withont changing the pattern of ventilation. The equipment must also allow the collection of pure expired gases for analysis and must be safe and reliable.

We have designed a ventilator which makes possible the measurement of changes in FRC, $\mathrm{VO}_{2}$, and pulmonary mechanics of anaesthetized children. Simplified mechanical details appear in Figure 1. The piston ( $P$ ) of a 1500 cc syringe moves back and forth under the infuence of compressed air and vacuum, alternately supplied to the chamber behind the piston through solenoid valves SA and SV respectively.

The volume in the syringe at any instant is converted to a voltage signal by means of a multiturn potentiometer (POT) mechanically coupled to the shaft of the piston. Thus each volume ahead of the piston from $V_{n}$ to $V_{\max }$ is represented by a unique voltage signal from $E_{0}$ to $E_{\max }$. The reciprocating action of the piston is achieved by using this voltage signal to trigger the solenoid valves $\mathrm{SA}$ and $\mathrm{SV}$ at the appropriate times.

For example, when solenoid $\mathrm{SV}$ is open, the piston moves backwards under the influence of the vacuum, until the volume in front of it reaches $V_{1}$. At this point the voltage signal $\mathrm{E}_{1}$ triggers an electronic switch to close solenoid $\mathrm{SV}$ and the piston stops. After a predetermined delay solenoid SA opens and the compressed air pushes the piston forward until $V_{u}$ is reached. At this point the voltage $\mathbf{E}_{0}$ triggers solenoid SA to close and the piston stops. After another predetermined delay solenoid SV opens again, the piston moves back, and the cycle is repeated. The volume delivered by the syringe per cycle is $V_{1}-V_{0}$ but since $V_{n}$ is usually set to correspond to zero volume, the tidal volume is simply $V_{1}$.

Figure 2 shows the mair mechanical details of the ventilator. The patient is ventilated through the patient port (PP) with the help of solenoid valves IS and ES, which are activated in symchronism with the ventilator. The rate of motion of

-From the Department of Anaesthesia, The Research Institute, The Hospital for Sick Children, Toronto, Ontario, Canada. 


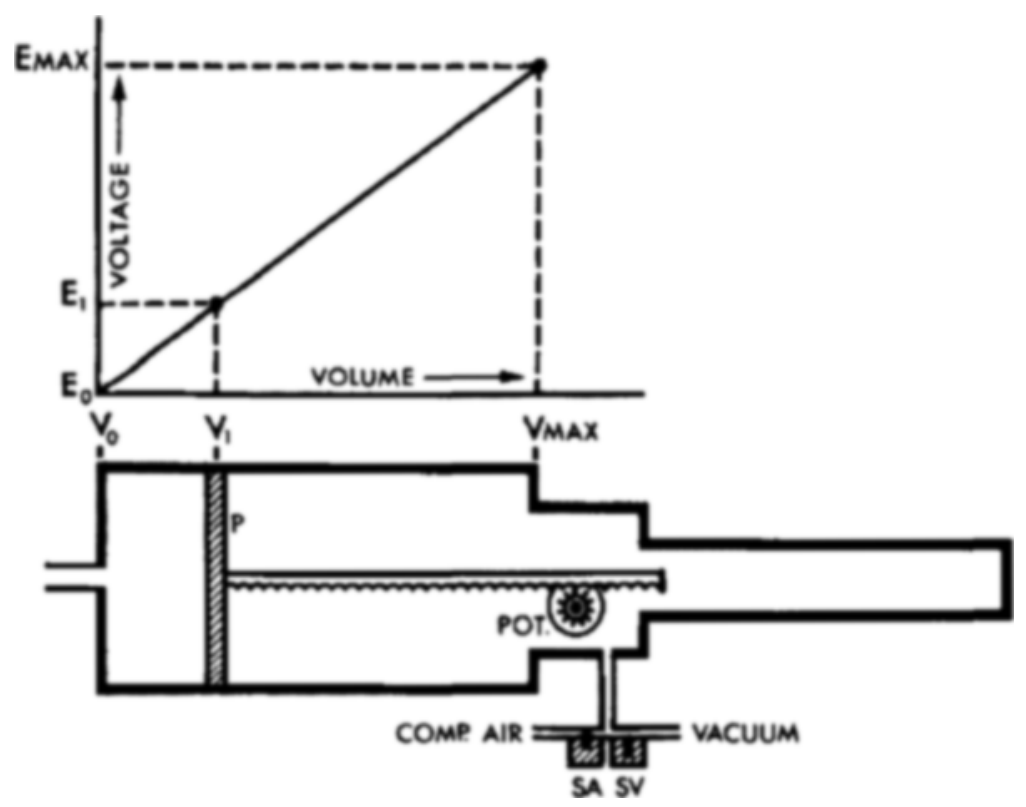

Frgure 1. Diagram showing correlation between volune (V) in front of the ventilator piston and voltage signal (E); solenoid valves for cornpressed air (SA) and vacuum (SV); potentiometer (POT).

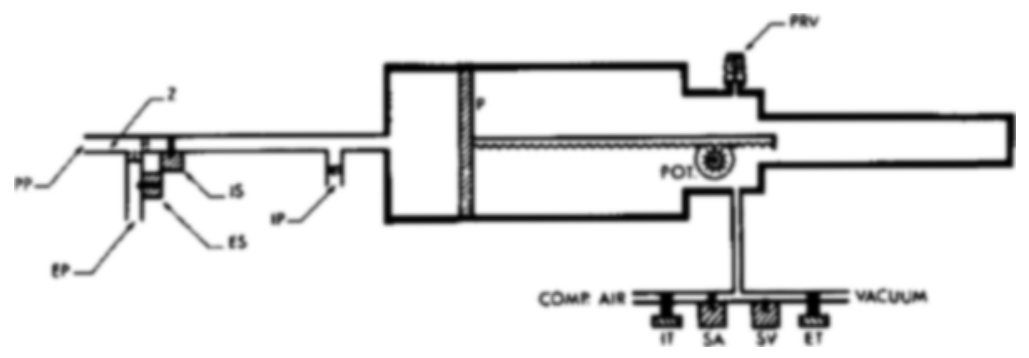

Fagure 2. Diagram showing mechanical details of ventilator: needle valves - inspiratory time control (IT) and expiratory time control (ET); pressure release valve (PRV); patient port (PP); expiratory port (EP); inlet port (IP); inspiratory solenoid (IS) and expiratory solenvid (ES); deadspace (Z).

the piston forwards and backwards is controlled by needle valves IT and ET respectively.

Figure 3 is a typical tracing demonstrating constant inspiratory flow, top band, and expiratory delay (breath hold), band three. Tidal volume, inspiratory flow, inspiratory and expiratory delays can each be altered independently of the others 


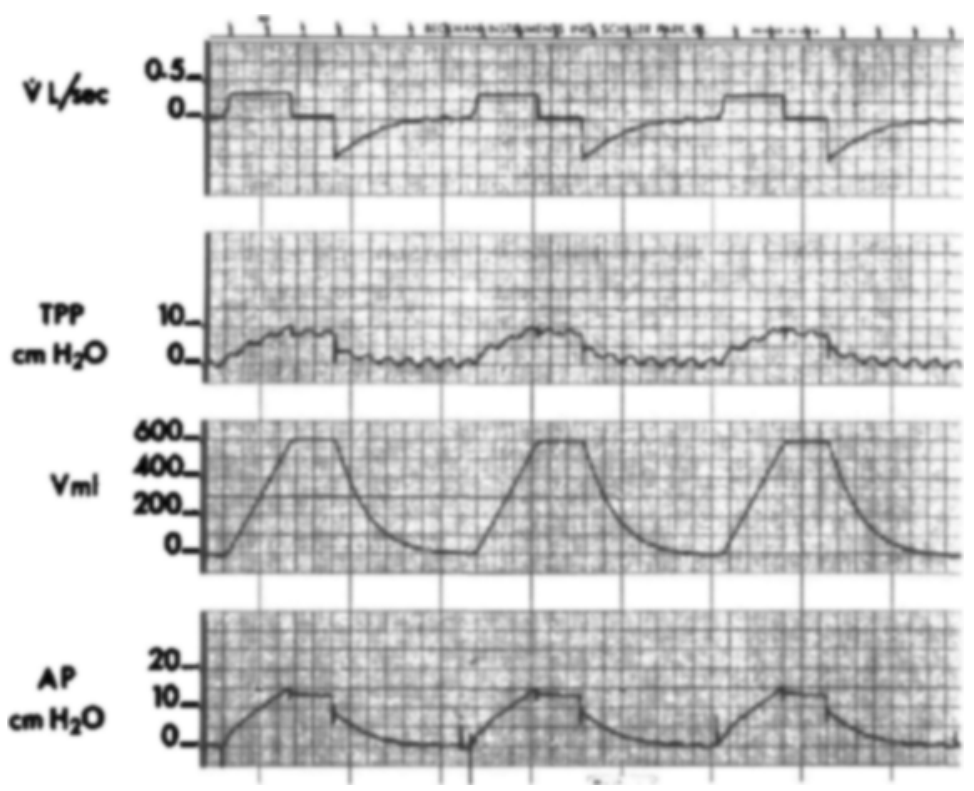

Ficure 3. Typical tracing showing constant inspiratory flow, transpulmonary pressure (TPP) tidal volume $(V)$ airway pressure and expiratory delay or "breath hold" phase (flat tops on the tidal volume trace).

while maintaining ventilation. For example, Figure 4 shows that inspiratory flow, the top band, can be increased, while the tidal volume, band three, is kept constant and Figure $\mathbf{5}$ shows that the tidal volume, band three, can be increased while the inspiratory flow, the top band, is kept constant.

A simplified diagram showing the triggering arrangement for these solenoid valves is shown in Figure 6. Each time the voltage signal equals $E_{0}$ or $E_{1}$ the polarity at the output of the trigger circuit reverses.

Assume the piston is moving backwards. The positions of the relay contacts and the polarity at the output of the trigger circuit are as shown in the figure. When the piston reaches $V_{1}$ the voltage signal equals $E_{1}$, the polarity at the output of the trigger circuit reverses and relay $T_{1}$ is energized. At this point the contact $P$ jumps to position $\mathrm{B}$, solenoid $\mathrm{SV}$ is turned off and the piston stops. Relay $\mathrm{T}_{2}$ is energized after a time delay determined by the setting of the inspiratory delay control (IDC). Then contact $R$ jumps to position $B$ enabling solenoid $S A$ to turn on and the compressed air moves the piston forward.

When the piston reaches $V_{0}$, which is usually set at the end point of its travel, the voltage signal becomes cqual to $E_{0}$, the polarity at the output of the trigger circuit reverses. When this occurs the relay $T_{1}$ de-energizes, contact $P$ jumps back to position A, solenoid SA closes, cutting off the supply of compressed air, and the piston stops. After a time delay, determined by the expiratory delay control (EDC), 


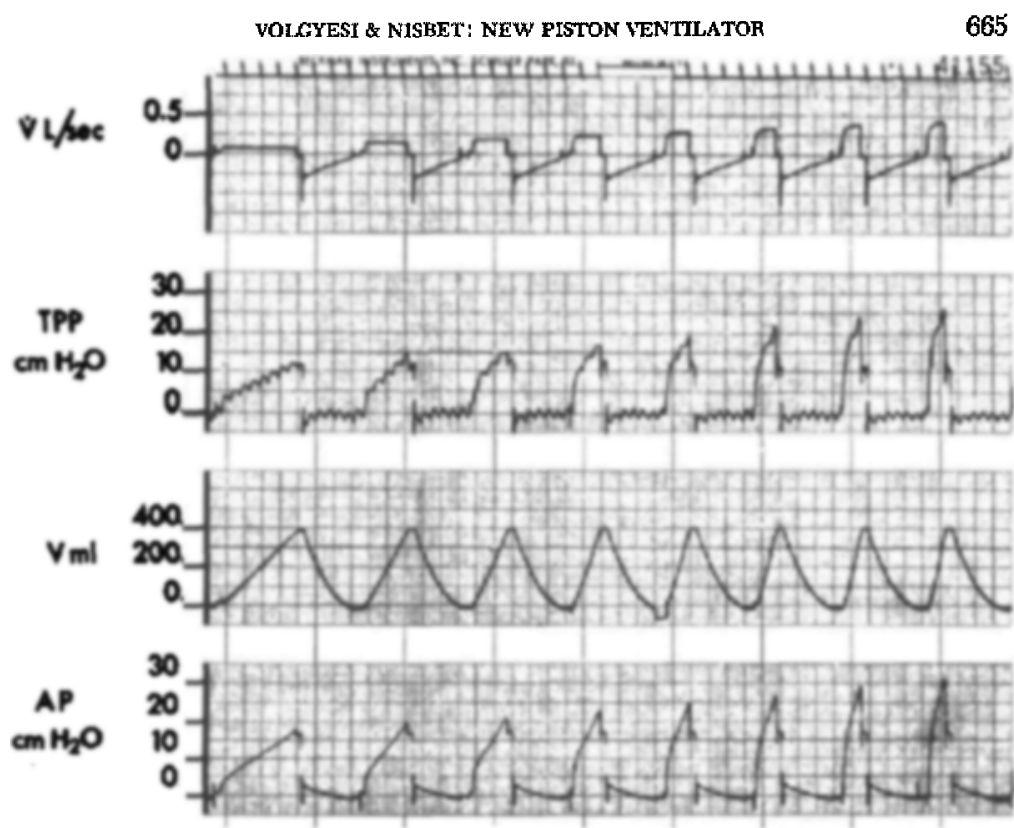

FIcure 4. Typical tracing showing increased jnspiratory flow (V) with no change in tidal volume (V).

$\mathrm{T}_{2}$ also de-cnergizes, contact $\mathrm{P}$ jumps back to position $A$ turning solenoid $\mathrm{SV}$ on, the piston moves backwards pulled by the vacuum, and the cycle repeats.

\section{Ventilation on open circuit}

With clamps at point $L$ and $N$ (Figure 7) the patient is on open circuit ventilation. Fresh gas enters the reservoir bag (RB) at the fresh gas port (FG). During the inspiratory phase the inspiratory solenoid (IS) is open, the expiratory solenoid (ES) is closed and the piston is driven forward, pushing the gas mixture into the patient at the patient port (PP).

During the expiratory phase the inspiratory solenoid (IS) is closed, the expiratory solenoid (ES) is open, and expired gas from the patient is vented through the expiratory port (EP) to the atmosphere or to a rubber bag if gas collection is required. During the same phase the piston $(P)$ moves backward, refilling the ventilator with fresh gases from the reservoir bag (RB).

\section{Ventilation on closed circuit}

The patient can be ventilated by closed circuit for the measurement of FRC by placing clamps at points $K, M$, and $O$.

During the inspiratory phase the inspiratory solenoid (IS) is open, the expiratory solenoid (ES) is closed, and the piston is driven forward, pushing the gas mixture into the patient at the patient port (PP). 


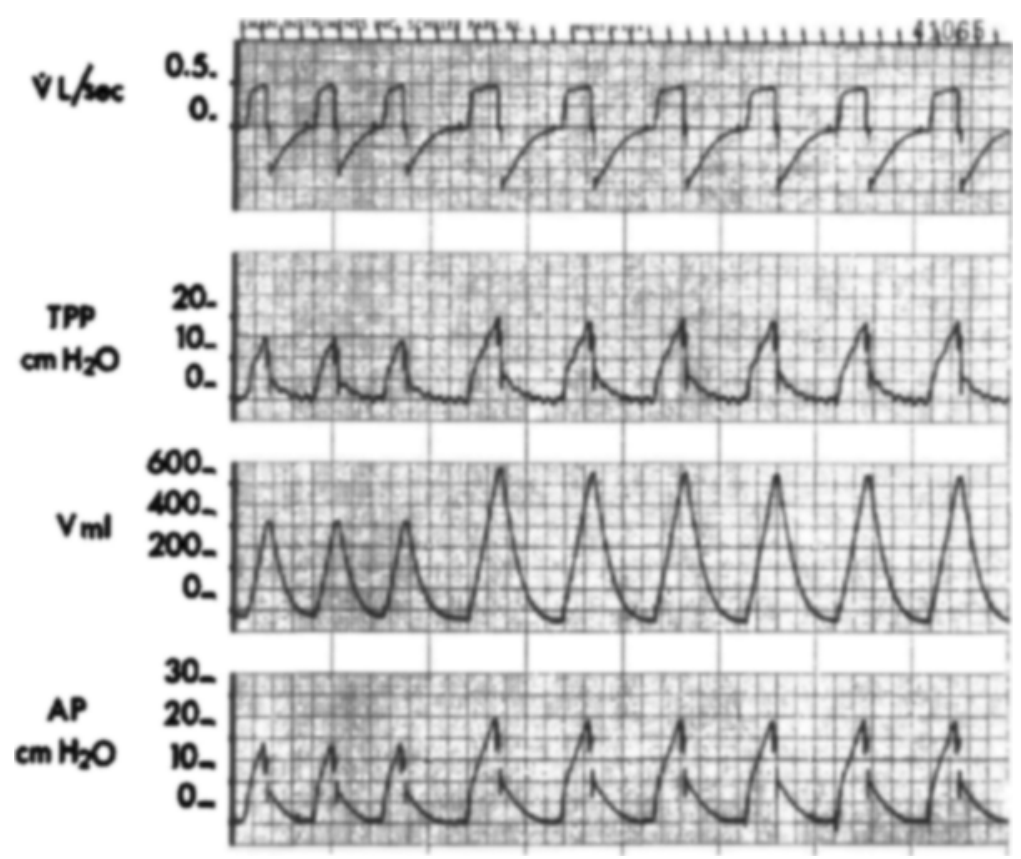

FIGUas 5. Typical tracing showing increased tidal volume (V) with no change in inspiratory flow ( $V$ ).

During the expiratory phase the inspiratory solenoid (IS) is closed, the expirntory solenoid (ES) is open and expired gas from the patient goes through the $\mathrm{CO}$. absorber $(A B)$ where $C O$ is removed, then through a circulating pump (CP) into the spirometer at $\mathrm{E}$. During the same phase the piston $(P)$ is drawn back and the ventilator refills with fresh gases from the spirometer at $D$.

Oxygen to replace the amount laken up by the patient is fed continuously into the circuit at $O_{2 .}$. The circulating pump sends a portion of the gases in the circuit through the helium analyzer to monitor helium concentration continuously without volume loss. Methoxyflurane affects the helium analyzer, so silica gel is placed in the intake line of the analyzer to adsorb it. To counteract negative pressure in the endotracheal tube produccd by the circulating pump at the end of expiration, a weight is plased on the spirometer bell. The system can then be balanced by vary. ing the speed of the circulating pump until the endotracheal pressure at the end of expiration is atmospheric.

\section{FRC measurement}

With the patient ventilated on open circuit, the spirometer, $\mathrm{CO}_{2}$ absorber, circulating pump, helium analyzer and the connecting tubing axe flushed with the same gas mixture the patient is breathing, and are then isolated by placing clamps 


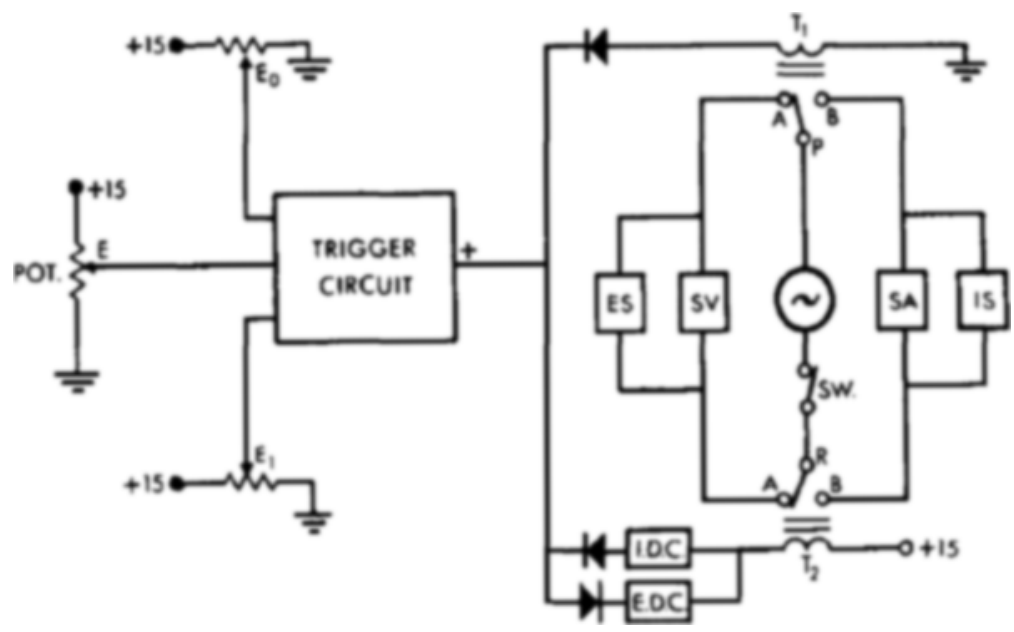

FIGure 6. Simplified diagram of ventilator circuitry showing triggering mechanism for solenoids (ES, SV, SA, IS); inspiratosy delay ontrol (IDC) and expiratory delay control (EDC); relays $\left(T_{1}, T_{2}\right)$; stop switch $(S W)$.

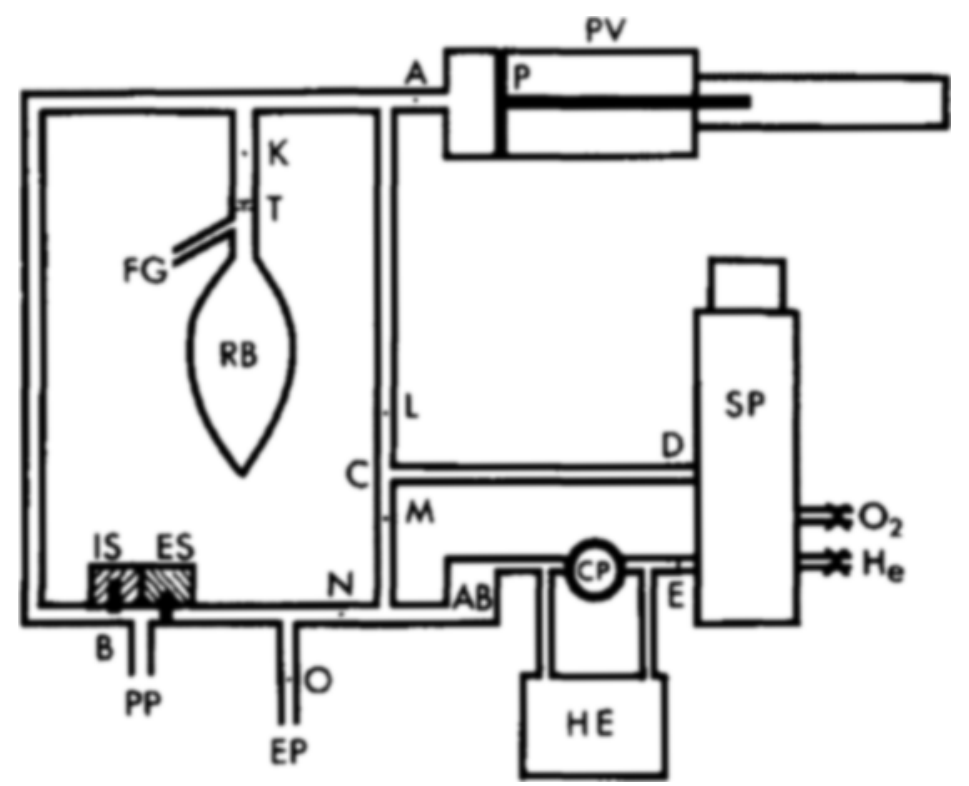

Freure 7. Diagram showing complete respiratory circle. Piston ventilator (PV); spirometer (SP); helium catharometer (HE); circulating pump (CP); carbon dioxide absorber ( $A B$ ): reservoir bag ( $\mathrm{RB}$ ); fresh gas inlet ( $F G$ ); clamping sites $(K, L, M, N, O$ ). 
at points $\mathrm{L}$ and $\mathrm{N}$, A known amount of helium is then introduced into the small circuit so produced by way of the He inlet and the initial concentration is recorded.

To measure FRC, the piston is first stopped at its zero volume position and a clamp is placed at point $K$. Then the clamp at $L$ is removed and the piston is drawn back, filling the syringe from the spirometer, while the patient expires passively through the expiratory port (EP) to atmosphere. When the volume in the ventilator equals the tidal volume, the piston is stopped and a clamp is placed at point $L$. The clamp at $\mathbf{N}$ is then removed and the ventilator is set in motion, ventilating the patient on closed circuit.

The total volume changes from one cycle to the other, observed on the spirometer trace, can be reduced to zero by introducing oxygen at $\mathrm{O}_{2}$ to balance the oxygen uptake of the patient. When the rate of oxygen uptake is balanced by the inflow and the helium concentration in the circuit has become steady, the helium concentration is recorded and the patient is switched back to the open circuit. The FRC of the patient can then be calculated using the formula based on the volume dilution of helium. ${ }^{1}$

\section{Expired gas collection}

To collect expired gases uncontaminated by inspired gases is more difficult from children than from adults because a fixed amount of inspiratory gas dissolved in a small volume of expired gas causes more contamination than it would if dissolved in a large volume of expired gas. To keep errors due to contamination within acceptable limits, the smaller anatomical deadspaces and tidal volumes of children require smaller apparatus deadspace and more effective separation of inspiratory and expiratory gases than adults.

To meet these demands we also designed a breathing valve to be used with the ventilator. Effective gas separation is achieved by triggering two solenoid valves (IS and ES) on and off in synchronism with the ventilator (Figure 2). Triggering is arranged in such a way that one valve closes before the other one opens, so that both valves are never open at the same time. To ensure mirimum deadspace, two one-way rubber flap valves are also included, one in each limb as close to the patient port (PP) as physically possible. With such an arrangement, the only place where inspiratory and expiratory gases mix is in chamber $\mathrm{Z}$ (Figure 2), which can be kept small compared to the anatomical deadspace of the patient.

\section{Safety features}

The possibility of accidental over-inflation of the Jungs is prevented by the pressure release valve (PRV in Figure 2), which is adjustable to a pressure slightly above the maximum value expected during normal ventilation of the patient. If for any reason the pressure rises above this value, the compressed air spills through the PRV and the piston stops. In the case of power failure the piston stops because the solenoid valves used to activate the ventilator are open only when energized.

The ventilator can also be stopped at any time manually by the stop switch (SW in Figure 6), which cuts off the power to the solenoids, leaving the power for the rest of the electronic section on. This ensures that the ventilator can be reactivated from the point on the respiratory cycle at which it was stopped. Since minute venti- 
lation can be accurately controlled using this ventilator the chances of over- or under-ventilating the patient are small. All the exposed metal parts, including the solenoid valves, are properly grounded and the patency of the ground connections is checked routinely to avoid any danger of electric shock.

The ventilator in its present form should not be used where explosive anaesthetic agents are being administered.

\section{Discussion}

Although the ventilator described above is primarily a research tool, it has several advantages which make it desirable for clinical practice:

(1) It is simple to operate.

(2) It can provide constant tidal volume for long periods of time in spite of any changes in compliance or resistance.

(3) It makes possible changes in tidal volume, inspiratory and expiratory times and delays independently of each other.

(4) It is able to provide a "breath hold" phase at the end of inspiration which may be useful in ventilating patients with respiratory problems.

(5) It is rugged and reliable because it has few moving parts.

(6) It is inexpensive to build and to maintain.

\section{ACKNOWLEDGMENTS}

We gratefully acknowledge the assistance of Dr. T. L. Dobbinson who has done a tremendous amount of work both in the laboratory and in the operating rooms testing, modifying, and evaluating the equipment. We are also grateful to those members of the Department of Anaesthesia at The Hospital for Sick Children who have made this work possible.

\section{REFERENCE}

I. Donninson, T.L., Nrsbet, H.l.A. \& Pelton, D.A. Functional residual capacity (FRC) and compliance in anaesthetized paralyzed children. Part I: Methods (in preparation). 